\title{
Role of shape and localized resonances in extraordinary transmission through periodic arrays of subwavelength holes: Experiment and theory
}

\author{
K. L. van der Molen,,${ }^{1} *$ K. J. Klein Koerkamp, ${ }^{1}$ S. Enoch,${ }^{2}$ F. B. Segerink, ${ }^{1}$ N. F. van Hulst, ${ }^{1}$ and L. Kuipers ${ }^{1,3, \dagger}$ \\ ${ }^{1}$ Applied Optics Group, Department of Science \& Technology and MESA ${ }^{+}$Research Institute, University of Twente, P.O. Box 217, 7500 \\ AE Enschede, The Netherlands \\ ${ }^{2}$ Institut Fresnel, CNRS 6133, Campus de St. Jérôme, case 161, 13397 Marseille Cedex 20, France \\ ${ }^{3}$ FOM-institute for Atomic and Molecular Physics (AMOLF), Kruislaan 407, 1098 SJ Amsterdam, The Netherlands
}

(Received 1 April 2005; published 12 July 2005)

\begin{abstract}
The effect of the aspect ratio of rectangular holes on the transmissivity of periodic arrays of subwavelength holes in optically thick metal films is investigated. The transmissivity is found to be highly dependent on the aspect ratio of the holes. Moreover, the wavelengths of maximum transmissivity show a monotonous shift as a function of the aspect ratio of the holes. We attribute the enhanced transmission of the periodic arrays to an interplay of surface plasmons at the surface of the metal and shape resonances (also known as localized modes) inside the holes. The importance of the shape resonances was confirmed by a comparison of transmission through periodic hole arrays and through randomly distributed holes. Dispersion curves of periodic and random hole arrays confirmed the existence of shape resonance as well. We suggest that the localized modes effectively act as waveguides and increase the coupling efficiency of surface plasmons between both sides of the film, which results in a higher transmissivity. The shift of the maxima of the transmissivity may in part be explained by the spectral position of the localized modes in the individual holes. Finally measurements on similar patterns in $\mathrm{Ni}$ and $\mathrm{Ag}$ revealed that the occurrence of shape resonances is independent of the material of the film.

DOI: 10.1103/PhysRevB.72.045421

PACS number(s): 81.07.-b, 42.79.Dj, 71.36.+c, 78.66.Bz
\end{abstract}

\section{INTRODUCTION}

The phenomenon of extraordinary transmission through periodic arrays of subwavelength holes in metallic films has received tremendous attention since its discovery in 1998 by Ebbesen and co-workers. ${ }^{1}$ In this phenomenon the transmitted fraction of the incident light can exceed the open fraction of the array for certain wavelengths. The phenomenon has also been called enhanced or resonant transmission and exhibits similarities to the transmission behavior of frequencyselective surfaces for frequencies ranging from the near infrared to those of microwaves. ${ }^{2,3}$ The large transmission has the potential for many applications. In addition, the underlying mechanism for the transmission itself is intricate and merits scientific attention.

The extraordinary transmission phenomenon is generally attributed to resonances of surface plasmon polaritons (SPP's) set up by the periodicity of the hole array (see, e.g., Ref. 4). Nevertheless, this interpretation is not without critique: it was pointed out that SPP's would actually be detrimental to the transmission. ${ }^{5}$ Although this is true in some specific cases, it turned out to be an invalid critique for the general phenomenon of transmission through periodic arrays of subwavelength holes. However, an explanation has recently been put forward that does not require the excitation of any SPP. ${ }^{6}$ To fully unravel the principles underlying the phenomenon it is important to investigate the effect of many contributing factors. Model calculations have elucidated the role of the thickness of the metal film, the periodicity and the type of metal. ${ }^{7,8}$ Other calculations have shown that localized modes can also contribute to the transmission. ${ }^{9,10}$ Proposals have been made to boost the transmission by using annular structures that actually contain propagative modes despite the fact that their size is subwavelength, ${ }^{11}$ which have recently led to the first experimental realization thereof. ${ }^{12}$

Experimentally, the role of periodicity, film thickness and metal type have been clarified. ${ }^{13-16}$ It has also been shown that increasing the hole size, increases the transmission. In particular for small hole sizes the increase as a function of hole size is much stronger than may be expected based on conventional aperture theory. ${ }^{17}$ This finding is consistent with careful studies of the radiative damping in the hole arrays,${ }^{18}$ which is shown to follow a Bethe-Bouwkamp power-law behavior. ${ }^{19}$ Recently, experiments and calculations have shown that changing the hole shape can greatly enhance the transmission through the films while inducing a red shift of the maximally transmitted peaks, ${ }^{20}$ a finding that was recently confirmed. ${ }^{21}$ This behavior cannot easily be explained in terms of surface plasmons only. Hole shape, and in particular the aspect ratio of rectangular or elliptical holes in the plane of the metal film also greatly affects the polarization properties of the structures, ${ }^{20,22-24}$ even in the TeraHertz regime. $^{25}$ In the optical regime the increased transmission associated with an increased aspect ratio of the hole shape has been attributed in part to localized modes (shape resonances). ${ }^{20,21}$ However, the investigations on the role of hole shape have not yet elucidated the role of the type of metal, crucial for determining the transmission mechanism. Moreover, only normal incidence measurements were reported so that no insight was gained on the dispersion properties of extraordinary transmission through arrays of shaped holes.

Here, we present a detailed analysis, consisting of both experiments and model calculations, of the effect of hole 
shape on extraordinary transmission. We will discuss its effect on polarization and on the wavelengths of the transmission peaks. Furthermore, the role of the metal is addressed: for optically thick films of $\mathrm{Au}, \mathrm{Ag}$, and $\mathrm{Ni}$, we have varied the aspect ratio of rectangular holes while keeping their area and the period of their lattice constant. We complete our study with an investigation of the dispersion of the transmission through the arrays. It is shown that hole shape greatly affects extraordinary transmission. By increasing the aspect ratio of rectangular holes the height of the transmission maxima can be increased, huge polarization anisotropy can be induced, and the peaks can be spectrally shifted to a large extent. We show that this general behavior is independent of the type of metal. The type of metal does, however, affect the extent of the transmission increase and the width of the peaks. The dispersion characteristics of the arrays are indicative of the strong influence of localized modes on the phenomenon of extraordinary transmission.

\section{EXPERIMENTAL}

The basis for all our samples was a metal film evaporated on a 1-mm-thick glass substrate. Measurements were performed on three different metals: $\mathrm{Au}, \mathrm{Ag}$, and Ni. These metals were chosen because the propagation length of surface plasmons on the three metals is significantly different for the optical frequencies used in this investigation. All film thicknesses were $215 \pm 15 \mathrm{~nm}$. The skin depth is roughly equal for all metals: approximately $30 \mathrm{~nm}$ in the relevant part of the spectrum. For all metals the film thickness is thus much larger than the skin depth. Coupling between surface plasmons from both sides of the film directly through the metal will therefore be negligible. The damping of surface plasmons is largest in $\mathrm{Ni}$ and smallest for $\mathrm{Ag}$, this is especially true for long wavelengths. Accordingly, the surface plasmon propagation length along the surface of the metal is therefore shorter in $\mathrm{Ni}$ than in $\mathrm{Au}$ and much shorter than in Ag.

A FEI Vectra 200 focused ion beam (FIB) was used to mill holes in the metal film. We milled arrays of $20 \times 20$ holes with a period of $425 \mathrm{~nm}$. This rather small value for the period was chosen because experiments have shown that the height of the transmission increases with the number of holes per area. ${ }^{15}$ While keeping the hole lattice constant, we changed the hole shape. We fabricated various arrays consisting of rectangular holes. In each array the aspect ratio of the rectangles was different. In Fig. 1 FIB images of square and rectangular hole arrays are shown. From these images it can be seen that the shape of the holes can be defined accurately and that the shapes of the holes are highly uniform.

In order to relate our results for the ordered arrays to the properties of individual holes, samples were fabricated with patterns in which the holes are not placed in a periodic array. 500 holes were randomly positioned within an area of 20 $\times 20 \mu \mathrm{m}^{2}$. These "random" hole arrays were fabricated so that the relative positions of the holes were equal for arrays containing different hole shapes.

Measurements were performed by illuminating the sample with normally incident light from a halogen lamp. This light source has a stable, high output power and a broad emission

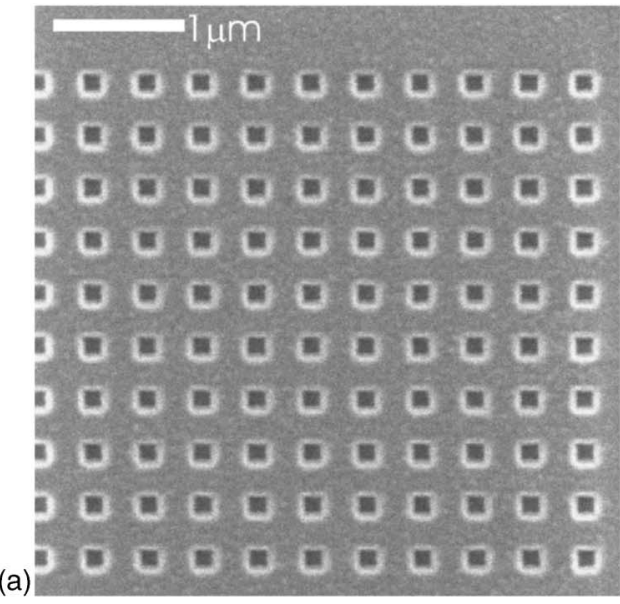

(b)

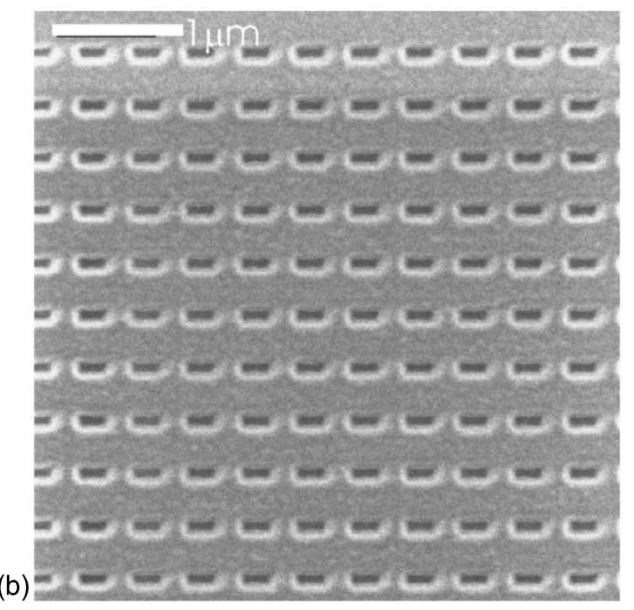

FIG. 1. Focused ion beam images of two periodic arrays: (a) square holes with an aspect ratio of 1 (size: $150 \times 150 \mathrm{~nm}^{2}$ ) and (b) rectangular holes with an aspect ratio of 2 (size: $106 \times 212 \mathrm{~nm}^{2}$ ). Both structures are fabricated in a 200-nm-thin Au layer and have a periodicity of $425 \mathrm{~nm}$. The holes have sharp edges and are highly uniform.

spectrum. The incoming beam was slightly focused (numerical aperture $=0.07$ ). The transmission spectrum of the patterns was measured with an optical multichannel analyzer consisting of a Jobin Yvon CP200 spectrograph in combination with a Princeton Instruments LN/CCD-512TBK backilluminated CCD camera. This setup allows transmission measurements between 480 and $1000 \mathrm{~nm}$, with a spectral resolution of $3 \mathrm{~nm}$. Liquid nitrogen cooling of the CCD camera reduced the background signal and enabled the detection of very low signals through long integration times. Integration times were controlled with a shutter in front of the spectrograph and were typically in the order of $10 \mathrm{~s}$. In all measurements presented here, the light was incident from the air side of the sample. Measurements with light incident from the glass side were also performed, and gave the same results within the experimental error. For the dispersion measurements the sample was tilted with respect to the incident beam.

The measured spectra were normalized to arrive at a value that can be interpreted as the effective transmissivity of the holes in the array. It is in fact the transmission of the array 
divided by the air fraction of the array. The transmissivity is a useful quantity to compare transmission phenomena through hole arrays with varying hole shapes and hole sizes. In order to correct for the possibly wavelength-dependent transmission of the measurement setup, the transmissivity was calculated by dividing the measured spectra of the patterns by the transmission spectrum of a large calibration hole in the same sample. The size of the hole was $10 \times 10 \mu \mathrm{m}$, large enough to validate the assumption that the transmissivity of this hole is 1 . Corrections were made for the difference between the area of the calibration hole and the total area of the holes in the array. Because of diffraction effects, typically $10 \%$ of the light that emerges from the arrays and 3\% of the light that emerges from the reference hole is not collected by the objective that is used to focus the transmitted light on the spectrograph. Corrections for diffraction effects were made by calculating the diffraction from the finite sizes array or reference hole and taking into account the collection angle of the optical system behind the sample.

\section{RESULTS}

Figure 2 shows the transmissivity spectrum of rectangular hole arrays with different aspect ratio fabricated in an $\mathrm{Au}$ film. The area of the holes is kept constant for all arrays at a value of $22500 \mathrm{~nm}^{2}$. Figures 2(a) and 2(b) present the transmissivity for polarizations perpendicular ( $y$ polarization) and parallel ( $x$ polarization) to the long axis of the holes, respectively (see insets). Note the difference in scale on the $y$ axes. The transmission spectra are clearly affected by the incident polarization. The first effect that is immediately obvious as the aspect ratio of the holes is changed, is the change in transmissivity of the peaks. For $y$-polarized light the transmissivity increases strongly when the aspect ratio is enlarged. For the $(0,1)$ peak we observe an increase in transmissivity of more than an order of magnitude from 0.2 for an aspect ratio of 1 (square holes) to a value of 2.9 for an aspect ratio of 3. Interestingly, the increase of transmissivity is interrupted for the hole array consisting of holes with an aspect ratio of 2.5. This result has been reproduced for different hole arrays with the same aspect ratio. For the chosen hole area and array period, an aspect ratio of 2.5 results in hole widths in the $x$ direction that are roughly equal $(237 \mathrm{~nm}$ air vs $188 \mathrm{~nm}$ metal) to the length of metal between the holes. An increase in the aspect ratio leads to a decrease in transmissivity for $x$-polarized light. It is clear that the aspect ratio of the holes affects the amount of transmission and induces a strong polarization anisotropy.

In addition to the magnitude of the transmissivity, the positions of the peaks are also affected by the aspect ratio of the holes. The red shifts observed for the $y$ polarization will be discussed in detail. It is found that all peaks undergo a redshift. The $(0,2)$ peak shifts to longer wavelengths and coincides with the $(1,1)$ peak. The combined peak shifts to longer wavelengths as well. It seems that the red shift of this combined peak cannot proceed beyond the transmission minimum caused by the first order Wood's anomaly around $670 \mathrm{~nm},{ }^{26}$ which is largely unaffected by the aspect ratio of the holes. The position of the $(0,1)$ peak also undergoes large
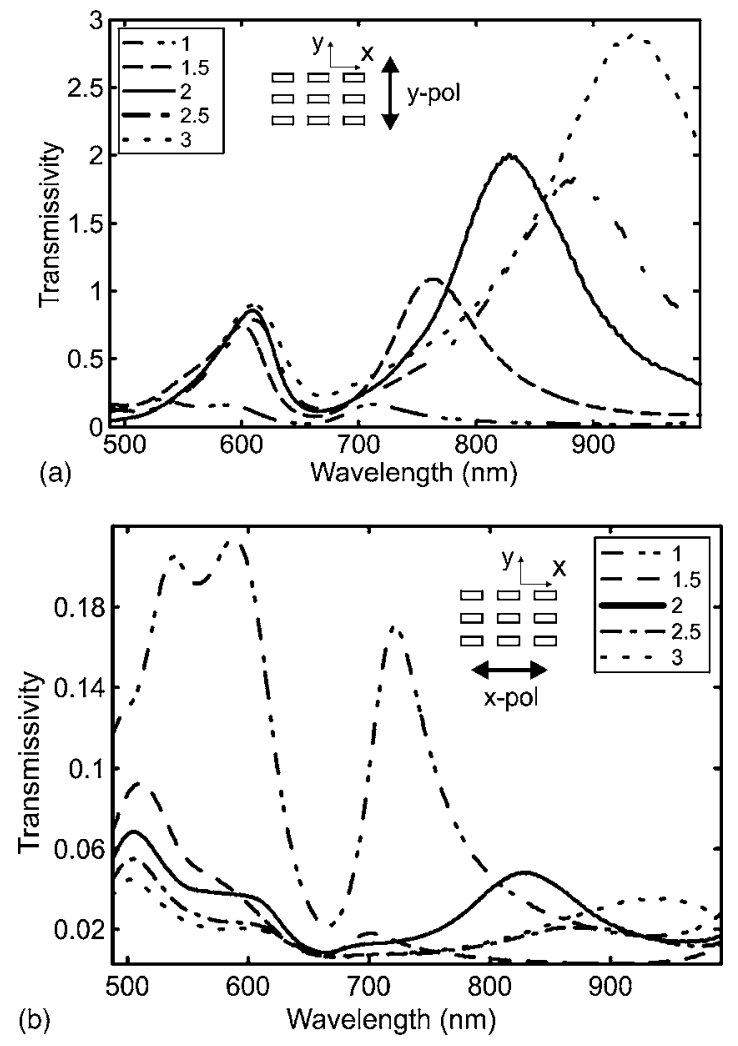

FIG. 2. The transmissivity of structures with different aspect ratio of the holes while keeping the hole area constant for two different polarizations of light. The legends show the aspect ratios of the holes. In the insets the used axes are displayed. (a) The transmissivity for $y$-polarized light shows an increase in transmissivity by an increase in aspect ratio. The peaks are broadened and shift to longer wavelengths. (b) For $x$-polarized light the opposite occurs: a decrease in aspect ratio leads to an increase in transmissivity.

spectral shifts to longer wavelengths. In Fig. 3 the position of the $(0,1)$ peak is plotted as function of the aspect ratio of the holes. The shift of the $(0,1)$ peak when the aspect ratio is increased from 1 to 4 is more than $200 \mathrm{~nm}$ and the shift depends linearly on the aspect ratio of the holes. The shift of the $(0,1)$ peak, excited with $x$ polarization, exhibits a differ-

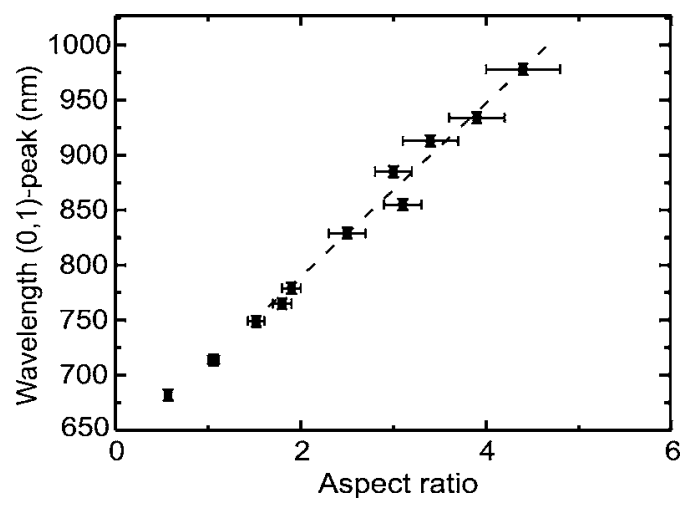

FIG. 3. The wavelength of the $(0,1)$ peak as a function of aspect ratio. The peak shifts monotonously to longer wavelengths when the aspect ratio increases. 

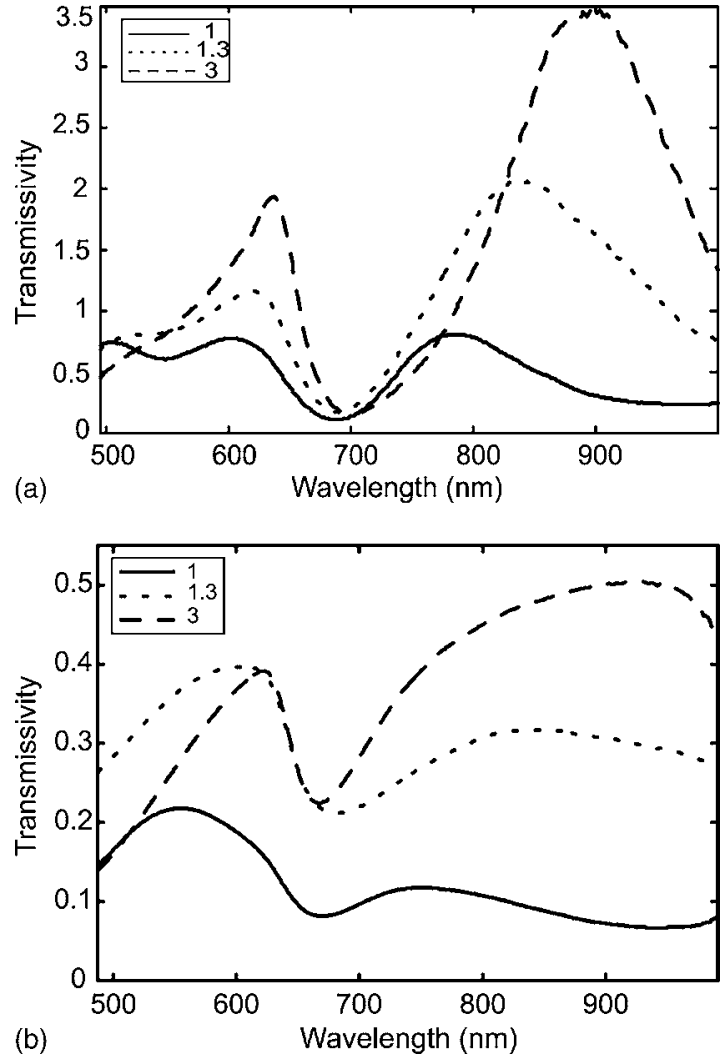

FIG. 4. (a) The transmissivity for square holes and two types of rectangular holes (aspect ratio 1.4 and 2.5, respectively) in a $230 \mathrm{~nm} \mathrm{Ag}$ layer. The transmissivity increases when the aspect ratio is larger and the peaks shift to longer wavelengths. (b) The transmissivity for square holes and two types of rectangular holes in a $220 \mathrm{~nm}$ Ni layer. The transmissivity increases when the aspect ratio is larger. The shift of the peaks is less clear, but still present.

ent behavior. An increase in the aspect ratio first leads to a small blueshift. The fact that for larger aspect ratios the behavior is identical to the $y$ polarization is attributed to a small deviation of the actual, experimental polarization with respect to the $y$ direction that leads to the transmission of a small part of the $(1,0)$ peak for the $x$ polarization.

In order to investigate the role of the type of metal three types of hole arrays are milled in Ag and Ni films: a square hole array and two rectangular hole arrays (with aspect ratios of 1.4 and 2.5, respectively). The period of the array is kept constant at $425 \mathrm{~nm}$. The results for the Ag film for $y$ polarized light are shown in Fig. 4(a). The transmission behavior as the hole shape is changed of the arrays made in a Ag film is clearly the same as the behavior observed for $\mathrm{Au}$ films. Again a large increase in transmissivity is observed as the aspect ratio becomes larger. The initial transmissivity is higher than the transmissivity of the Au film. We attribute this to the fact that the SPP's are less damped on Ag than on $\mathrm{Au}$. The maximum transmissivity for the highest aspect ratio holes increases by 2.8 with respect to that of the square holes to almost 3.5, which corresponds to an absolute transmission of $28 \%$. The redshift of the peaks is also apparent. It is noteworthy that the measured redshift of the $(0,1)$ peak when the aspect ratio is changed from 1 to 2.5 is reasonably similar for

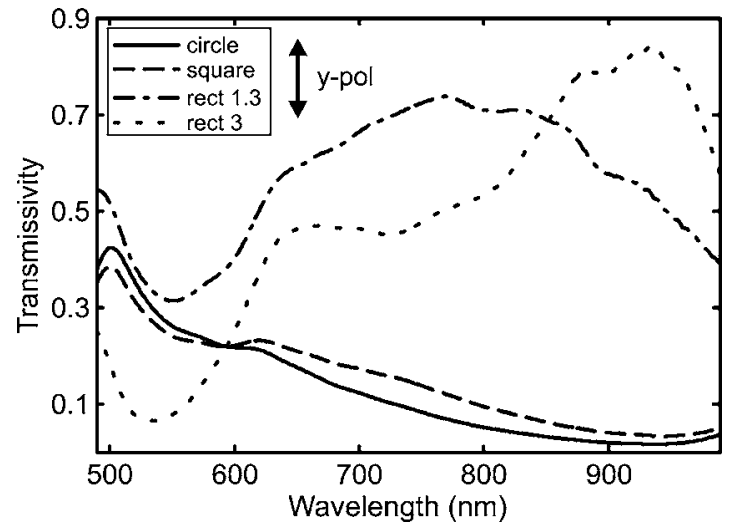

FIG. 5. The transmissivity of random hole arrays in Au for three forms: circles with a diameter of $190 \mathrm{~nm}$, squares (170 $\times 170 \mathrm{~nm}^{2}$ ), and two types of rectangles with an aspect ratio of 2:3 $\left(150 \times 225 \mathrm{~nm}^{2}\right)$ and of $1: 3\left(75 \times 225 \mathrm{~nm}^{2}\right)$. For increasing aspect ratio, the position of the peak shifts to longer wavelengths and the transmissivity increases.

$\mathrm{Au}$ and Ag: $120 \mathrm{~nm}$ for $\mathrm{Ag}$ and $170 \mathrm{~nm}$ for $\mathrm{Au}$.

Figure 4(b) shows how the transmissivity of an array fabricated in a $\mathrm{Ni}$ film is affected by the aspect ratio of the holes. As for $\mathrm{Au}$ and $\mathrm{Ag}$ the transmissivity increases when the aspect ratio is increased. Compared with the results in $\mathrm{Au}$ and Ag the peaks in the spectra are now much broader and have a lower maximum value. This can be directly related to the much higher damping of SPP's for Ni. Due to the large width of the peaks, the redshift is harder to determine than for the other two metals. Nevertheless, the size of the redshift for Ni arrays, $150 \mathrm{~nm}$, lies in between the redshifts observed for $\mathrm{Au}$ and $\mathrm{Ag}$. This indicates that the geometry of the holes is a dominant factor in the observed redshifts, whereas the optical properties of the metals seem to play a less important role where the peak position of rectangular hole arrays is concerned.

In order to investigate the role of hole shape in extraordinary transmission in greater detail, the transmissivity of individual holes is measured. For these measurements $\mathrm{Au}$ samples are investigated with a low density of randomly positioned holes (500 holes in an area of $20 \times 20 \mu \mathrm{m}^{2}$ ), in order to obtain a signal that is significantly larger than the residual transmission through a $200 \mathrm{~nm}$ film. For each random area the relative positions of the holes are identical while the shape of the holes is varied. The results of the measurements are presented in Fig. 5. While for circular and square holes the transmissivity roughly follows a BetheBouwkamp decrease with increasing wavelength (transmissivity $\propto \lambda^{-4}$ ), a strong enhanced transmission is observed for the rectangular holes. With increasing aspect ratio the centerof-mass of the transmission enhancement with respect to Bethe-Bouwkamp exhibits a redshift. Note that due to the random positioning the existence of surface plasmon resonances is minimal and can therefore not be the cause of the increase in transmissivity and the shift of the peaks. The lack of resonances was confirmed by the fact that the same random arrays fabricated in a Ni film showed the same behavior, despite the much stronger damping of surface plasmon polaritons. The enhanced transmission for the individual rect- 

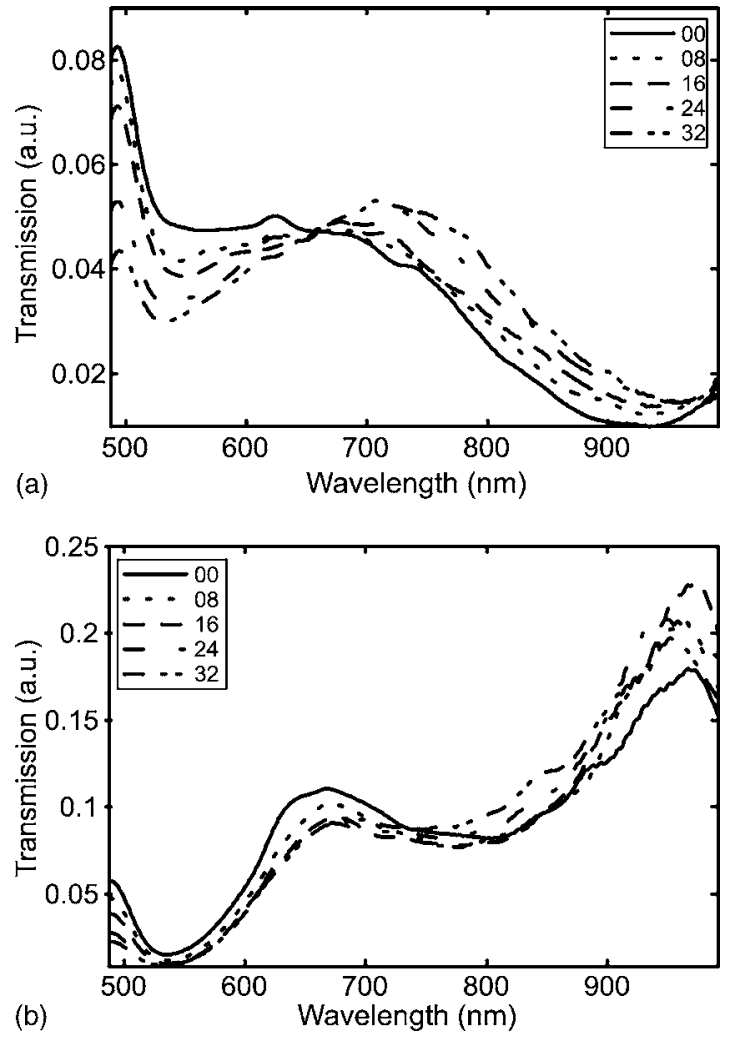

FIG. 6. (a) The normalized spectra for the random square hole is plotted for different angles of incidence. (b) The normalized spectra for random rectangle (aspect ratio 3) hole arrays are shown. The wavelengths of the maxima and minima stay almost at the same positions. Only for the squares a small shift can be seen of the peak around $700 \mathrm{~nm}$, a shift that is 8 times smaller than we measured for periodic hole arrays. In the legend the angle of incidence is listed.

angular holes can be attributed to localized modes (also called shape resonances) in the holes resulting from their shape. This also corroborates the observation that the redshifts observed in the hole arrays were dominated largely by the geometry of the holes and not by the type of metal.

All measurements presented so far were performed with the incident and emerging light normal to the sample. In effect this results in the measurement of the transmission for $k_{\|}=0$, where $k_{\|}$is the wave vector component of the incident light parallel to the sample surface. To measure the transmission for $k_{\|} \neq 0$, or in other words to measure the angular dispersion of the samples, the transmission of the arrays is measured as a function of the angle $\theta$ with respect to the optical axis of the measurement setup. In Figs. 6(a) and 6(b) the transmission spectra for different angles $\theta$ are presented for random arrays of squares $\left(170 \times 170 \mathrm{~nm}^{2}\right)$ and of rectangles $\left(225 \times 75 \mathrm{~nm}^{2}\right)$, respectively. The minute shift of $20 \mathrm{~nm}$ of the spectrum for the random array of square subwavelength holes when $\theta$ is changed from $0^{\circ}$ to $32^{\circ}$, shows that resonant behavior in the random arrays plays only a minor role. The shift of $20 \mathrm{~nm}$ corresponds to only 3 $\times 10^{-4} \mathrm{~nm}^{-1}$ in this wavelength range. The maxima and minima in the spectra of the random arrays of rectangles remain at the same wavelengths, which indicates that significant resonant behavior due to the relative positions of the

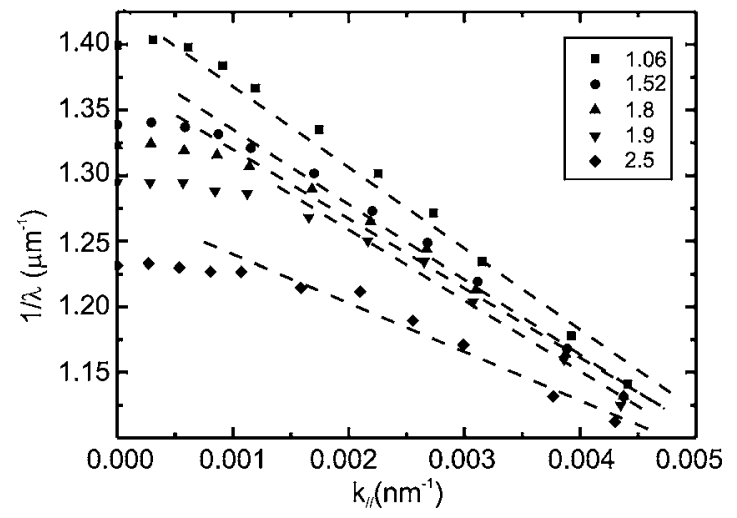

FIG. 7. The dispersion relation of the $(0,1)$ peak for different aspect ratios. The dispersion changes when the aspect ratio of the rectangular holes is increased. The fitting lines become less steep and around $\mathbf{k}_{\|}=0$ the dispersion relation becomes flatter. This indicates a stronger degree of localization in the transmission phenomenon.

holes is completely absent (despite the fact that these positions are identical to those of the random square hole arrays).

Dispersion measurements of the periodic hole arrays exhibit a very interesting behavior as the aspect ratio is varied for constant hole sizes. The results are presented in Fig. 7. Here, the dispersion of the $(0,1)$ peak is shown for different aspect ratios. The $1 / \lambda$ values at $k_{\|}=0$ decrease for higher aspect ratios, representing the redshift observed in Fig. 2(a). For large $k_{\|}$, i.e., larger than $0.002 \mathrm{~nm}^{-1}$ the dispersion of the peaks is similar to that of periodic arrays consisting of cylindrical subwavelength holes. ${ }^{4}$ However, for low $k_{\|}$values a strong effect of the aspect ratio of the rectangular holes is visible: with increasing aspect ratio the dispersion curves show an increased flattening. The flatness of the dispersion curves at low $k_{\|}$values is strong evidence for the highly localized nature of the modes that mediate the transmission. The degree of localization seems to increase with increasing aspect ratio.

\section{MODELING}

We also performed model calculations to investigate the role of hole shape on the extraordinary transmission. The aim of the following paragraph is to give to the reader the minimum of knowledge about the numerical method we use in order to clarify the model used. We refer the interested reader to the given references. Thereafter we describe the results of the calculations.

For the calculations we consider a biperiodic structure along the $x$ and $y$ axes, which is finite along the $z$ axis. We assume that the structure is illuminated by a plane wave with a wavelength $\lambda$. Thus, we consider a harmonic problem. Note that in our numerical simulations the optical indices of the materials (metals and substrate) are wavelength, i.e., frequency, dependent.

The numerical calculations of the transmitted light have been performed with the Fourier modal method that has been developed for the modeling of diffraction gratings. ${ }^{27,28}$ The method was only recently extended to biperiodic gratings 
due to the computational burden involved by the modeling of such three-dimensional structures. ${ }^{29}$ It is a modal method, i.e., the field is developed on a modal basis in each region of the space. In the precise case under study, it means that the electromagnetic field is developed on a plane wave basis in the substrate (in our case the glass) and the superstrate (the air) and on a modal basis dependent on the actual geometry in the metallic layer itself. The only hypothesis on the geometry of the problem is that in the grating structure (in the metallic layer) the optical index function must be invariant along the $z$ axis.

Subsequently, the boundary conditions at the limits of the metallic film are enforced. Note that these boundary conditions are conveniently contained in the $S$-matrix algorithm that we use and that also ensures the numerical stability of the code when the thickness of the grating becomes important. ${ }^{30}$ The problem is completed by a radiation condition that is equivalent to the Sommerfeld condition but now for grating problems. That is to say that the diffracted field (the total field minus the incident one) at infinity can be described as a superposition of outgoing plane waves, the orders of the grating. Precisely, the electric field in the three regions of space are given by

$$
\begin{aligned}
\mathbf{E}_{1}= & \mathbf{D}_{1}^{0} \exp \left(i \mathbf{k}_{\|}^{0} \cdot \mathbf{r}_{\|}-i k_{1, z^{0}}^{0}\right) \\
& +\sum_{n, m=-\infty}^{+\infty} \mathbf{U}_{1}^{n, m} \exp \left(i \mathbf{k}_{\|}^{n, m} \cdot \mathbf{r}_{\|}+i k_{1, z}^{n, m} z\right)
\end{aligned}
$$

in the superstrate, where $\mathbf{D}_{1}^{0}$ is the vector amplitude of the incident plane wave

$$
\mathbf{E}_{3}=\sum_{n, m=-\infty}^{+\infty} \mathbf{D}_{3}^{n, m} \exp \left(i \mathbf{k}_{\|}^{n, m} \cdot \mathbf{r}_{\|}+i k_{3, z}^{n, m} z\right)
$$

in the substrate and

$$
\mathbf{E}_{2}=\sum_{l=-\infty}^{+\infty} \mathbf{F}^{l}(x, y)\left[U_{2}^{l} \exp (i \gamma z)+D_{2}^{l} \exp (-i \gamma z)\right]
$$

in the metallic layer, with $\mathbf{k}_{\|}^{n, m}=\mathbf{k}_{\|}^{0}+n\left(2 \pi / d_{x}\right) \mathbf{e}_{x}$ $+m\left(2 \pi / d_{y}\right) \mathbf{e}_{y}$ and $\left(\omega^{2} / c^{2}\right) \nu_{i}^{2}=\left\|\mathbf{k}_{\|}^{n, m}\right\|^{2}+\left(k_{i, z}^{n, m}\right)^{2} . \mathbf{k}_{\|}^{0}$ is the tangential incident wave vector and $d_{x}$ and $d_{y}$ the periods along the $x$ and $y$ axes. A similar expression can be written for the magnetic fields.

The $S$ matrix associated to the problem links the diffracted field to the incident one

$$
\left[\begin{array}{c}
\mathbf{U}_{1}^{E} \\
\mathbf{U}_{1}^{H} \\
\mathbf{D}_{3}^{E} \\
\mathbf{D}_{3}^{H}
\end{array}\right]=\mathbf{S}\left[\begin{array}{l}
0 \\
0 \\
\mathbf{D}_{1}^{E} \\
\mathbf{D}_{1}^{H}
\end{array}\right],
$$

where $\mathbf{D}_{i}^{E, H}$ and $\mathbf{U}_{i}^{E, H}$ are vectors that contain both the $x$ and $y$ components of the Fourier developments [Eqs. (1) and (2)]
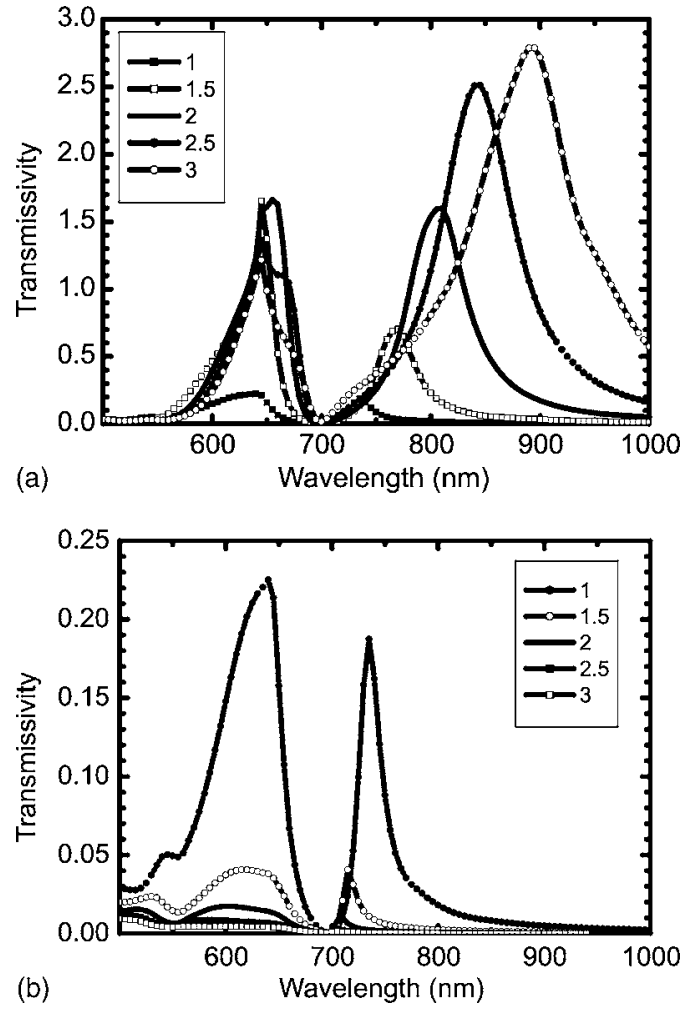

FIG. 8. Calculated transmissivity spectra for periodic hole arrays in a Au film with different aspect ratio (the surface area of the hole and the thickness are constant and identical to those used in the experiments). (a) Transmissivity for $y$-polarized light. (b) Transmissivity for $x$-polarized light

of the electric and magnetic fields (superscripts $E$ or $H$ ). The only non-null coefficients of $\mathbf{D}_{1}^{E}$ and $\mathbf{D}_{1}^{H}$ are those corresponding to the incident plane wave. The modal basis used in the metallic layer is obtained thanks to an eigenvector problem written in the Fourier space (this justifies the name given to the method) whose eigenvalues give us the propagation constants of the modes $\gamma$ and eigenvectors the field repartition of the modes $\mathbf{F}^{l}(x, y)$. We will not describe here the details, but in order to obtain accurate results one must pay attention to the way to write the products of Fourier series involved in this eigenvector problem. ${ }^{31}$ This method allows us to model any shape of the holes provided that the shape does not change along the $z$ axis and all the optical index of the materials can be considered as wavelength dependent. Note that the dispersion relation of the modes are obtained by searching for the poles of the scattering matrix of the system as usual. ${ }^{32}$

Figure 8 shows the computed transmissivity spectra for the periodic arrays in a Au film of rectangular holes for different aspect ratios. The geometrical parameters are identical to those given in the experimental section and the optical indices of the $\mathrm{Au}$ and glass substrate (BK7) are obtained from the literature. ${ }^{33}$ Note that the model does not take into account the finite thickness of the glass substrate and thus a difference with the experiments of about $4 \%$ is expected. The observed behavior is identical to the experimental trends. The large increase in the transmissivity with increasing aspect ratio, the very strong polarization anisotropy and the 

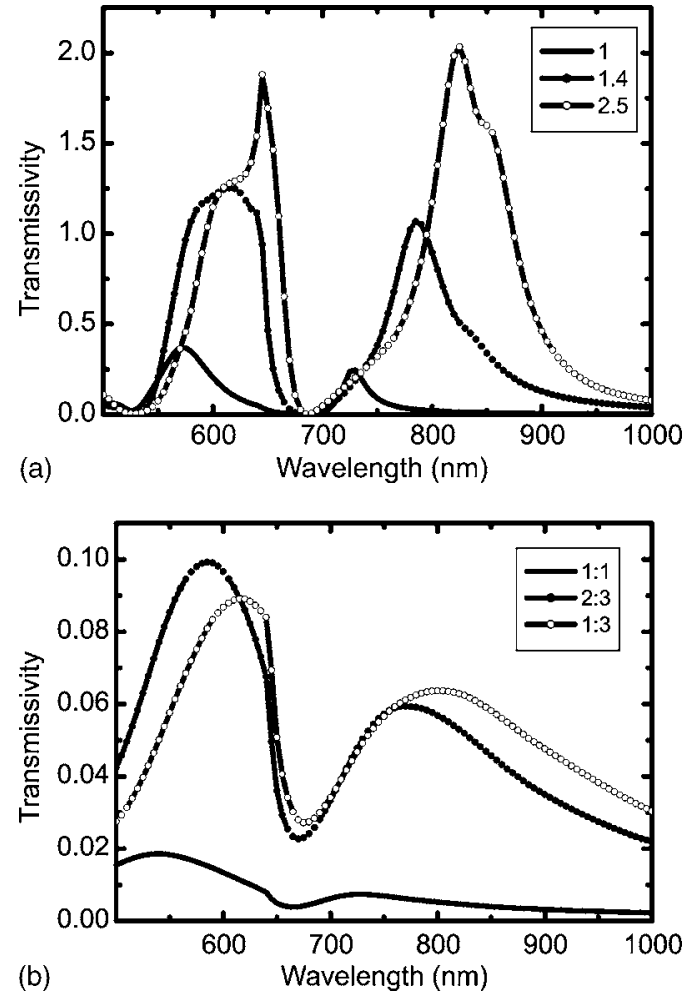

FIG. 9. Calculated transmissivity spectra for $y$-polarized light and for rectangular hole arrays with different aspect ratio. (a) Transmissivity through arrays in a Ag film. (b) Transmissivity through arrays in a Ni film.

redshift of the transmission are all reproduced. Note, that for incident $x$ polarization the $(1,0)$ peak exhibits a slight blueshift.

In Fig. 9 the transmissivity spectra of the Ag and Ni structures are shown. The optical indices of the metals are again taken from the literature. ${ }^{33}$ For these structures the redshifts are also observed and thus we confirm the crucial role of the shape of the hole.

The numerical and experimental results are in very good qualitative agreement for all curves. All the effects observed experimentally are confirmed by the modeling. It shows the relevance of the numerical method we use and that no important artifacts can be detected in the experiments. However, some discrepancies appear when one considers the level and exact shape of the transmissivity spectra. We attribute them mainly to the lack of precise knowledge of the optical indices of the metals. Indeed, it is well known that they can be affected by the deposition technique and that resonant effects can be strongly sensitive to their variations.

To gain more insight in the role of the holes shape, we have again considered Au structures on BK7 substrates. Now rather than the surface of the holes the length of one of the edges of the rectangular hole has been kept constant. In Fig. 10 (a), the $y$ dimension has been taken equal to $106 \mathrm{~nm}$ while the $x$ dimension varies. On the transmissivity spectra one can observe again the large redshift of the $(1,0)$ peak as already observed in Fig. 8. An increase of the value of the transmittivity is also obvious when the size increases, which was also already observed in Fig. 8 even when the hole area did not
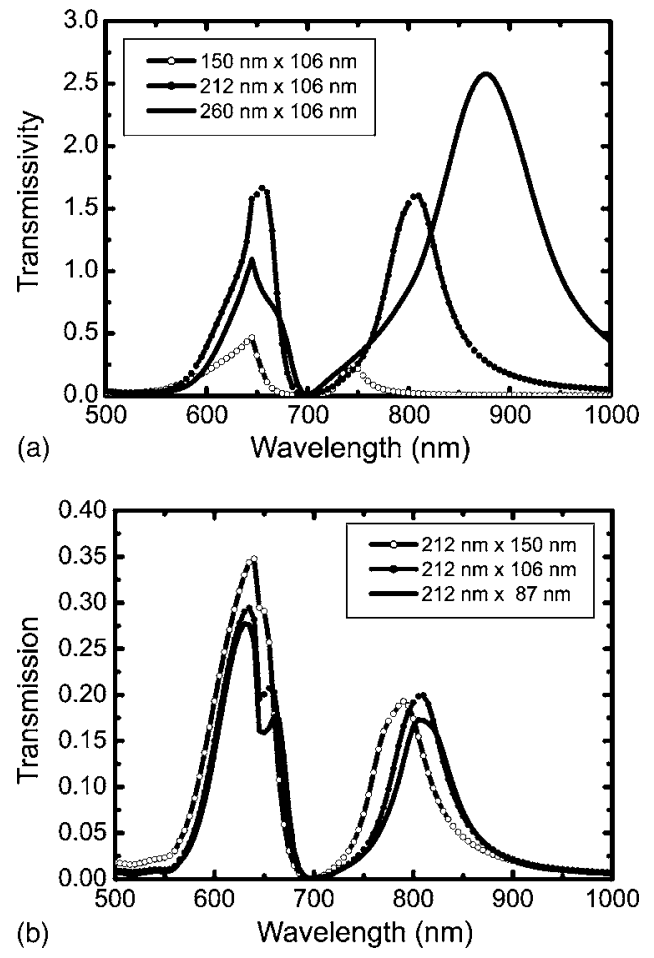

FIG. 10. Calculated transmissivity spectra for $y$-polarized incident light of arrays in a Au film in which the shape of the holes is varied by increasing only on side of the rectangles. (a) Hole side along $y$-direction constant. (b) Hole side along $x$-direction constant.

increase. In Fig. 10(b) we have represented the transmission spectra when the $x$ dimension is constant and the $y$ dimension varies. Note that, here, we have plotted the transmission rather than the transmissivity (i.e., not normalized by the surface ratio). From these spectra [Fig. 10(b)] two remarks arise. First, the amount of transmitted light is roughly the same for all the structures while the surface of the hole has been considerably changed. Second only a small redshift can be observed even when the size is decreased. Thus, we have shown evidence of the dramatic importance of the length of the $x$ side of the rectangular holes for a $y$-polarized incident field and of the different role played by each side of the rectangle.

In Fig. 11 the dispersion relation of electromagnetic modes are shown for two values of the aspect ratio. Only the $(1,0)$ peaks at the vicinity of the origin of the $k_{\|}$axis have been considered. These dispersion relations are obtained by minimization of the inverse of the determinant of the $S$ matrix defined by Eq. (4). The $(1,0)$ peaks observed on the transmissivity spectra are clearly obtained for the wavelength that correspond to the modes for $k_{\|}=0$. The effect of the shape of the hole on the dispersion of the mode is clearly shown in these figures: the larger aspect ratio, the flatter dispersion relation. It is clear that the redshifts on the transmissivity spectra are intrinsically linked to the flattening of the dispersion relation when the aspect ratio of the hole is increased.

\section{DISCUSSION}

Both our experiments and calculations show the large effect of hole shape on the phenomenon of extraordinary trans- 


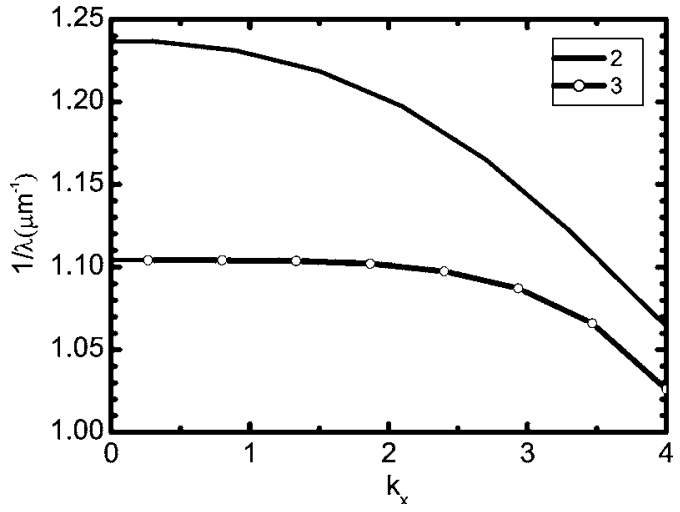

FIG. 11. Dispersion relation of the plasmon mode for rectangular hole arrays in a Au film for two different aspect ratios and a constant hole area. As the aspect ratio increases, so does the flat part of the dispersion close to $k_{\|}=0$, indicating an increase in the localized nature of the modes.

mission. By changing the aspect ratio of rectangular holes while keeping their surface area and the periodicity constant, we observe that many aspects of the transmission are affected. By varying the type of metal we have shown that the observed behavior is universal and does not depend on the type of metal. First of all, the transmission becomes highly dependent on the incident polarization. Light with an incident polarization perpendicular the long axis of the holes is transmitted several orders of magnitude better than light with a polarization along the long axis. This finding is consistent with experiments on elliptical holes ${ }^{22-24}$ although the degree of anisotropy reported here is roughly two orders of magnitude more. This quantitative difference is easily explained by the difference in film thickness used in the various experiments. As the tunneling probability depends on by how much the wavelength exceeds the cutoff condition, when considering the holes as waveguides, ${ }^{34}$ a certain polarization anisotropy for a given film thickness will be increased as the film thickness is increased.

Both the measurements on the isolated holes and the dispersion measurements on the periodic arrays indicate a strong role of localized modes, which increases with increasing aspect ratio. Note that work on the transmission through individual rectangular holes also suggests the existence of localized modes. ${ }^{35}$ Recent calculations of the properties of individual rectangular holes in a real metal film, indicate that decreasing the width of the holes induces a redshift of the cutoff condition. ${ }^{36}$ It has been recently suggested that localized modes play an important role only when their spectrum coincides with a peak in the extraordinary transmission spectrum that is caused by the periodicity of the array. ${ }^{21}$ This conclusion was drawn from experiments in which the period of the array was varied while the aspect ratio was kept fixed. However, our work shows that the full explanation is more complex: when we change the aspect ratio from 1 to 3 we observe that the $(1,0)$ peak actually shifts away from the position governed by the array periodicity (aspect ratio $=1$ ), while the transmission increases. Moreover, the transmission of the higher order peaks $(2,0)$ and $(1,1)$ also increases with increasing aspect ratio. From this we conclude that the red- shift and the enhancement of the transmission peaks in the periodic arrays, as the aspect ratio is increased, is affected by the resonance spectrum of individual holes, but the role of the periodicity of the array and the localized mode spectrum cannot be separated in a trivial manner. A suggestion to this effect was already made previously by us. ${ }^{20}$ Interestingly, no shape resonances were observed for rectangular holes in the $\mathrm{THz}$ regime. Neither did the spectra of the periodic arrays exhibit a redshift with aspect ratio in the $\mathrm{THz}$ regime. We suggest that this is a consequence of the smaller film thickness used in those experiments with respect to ours: $\lambda / 12$ and $\lambda / 4$, respectively. It is tempting to think that the rectangular holes support propagative modes, similar to those observed in the periodic annular holes. ${ }^{11,12}$ Note, however that for all aspect ratio's used in this investigation all the holes are smaller than the cutoff for all wavelengths used and all polarizations used. Nevertheless it is clear that the localized modes in the holes effectively do act as waveguides. ${ }^{10}$

It is interesting to note that our observations have strong parallels with observations made in scattering from nanoparticles and periodic arrays thereof. Of course, one has to be careful to invoke complementarity in the behavior of metals in the optical regime. Nevertheless, the similarities and the seeming complementarity are too striking to be left unmentioned. It is, for example, well known that metal nanoparticles exhibit localized plasmon resonances that depend on their shape. ${ }^{37,38}$ Interestingly, when a nanoparticle is elongated, the degeneracy due to the spherical symmetry of the excitation is lifted and two new modes appear, one along the long axis of the particle and one perpendicular to the axis. The longitudonal mode actually exhibits a redshift and also has a larger oscillator strength, ${ }^{38}$ whereas the transverse exhibits a smaller blueshift. This behavior is also evident in arrays of elongated particles in which coupling between the particles may be present. ${ }^{39}$ We observe the same behavior with one difference: the polarizations for the redshift and the blueshift are reversed. This would be consistent with the structures being complementary.

\section{CONCLUSIONS}

We have shown that the shape of individual holes in arrays of sub-wavelength holes in optically thick metal films has a dramatic effect on the optical transmission through the films. A significantly higher transmissivity was found when the aspect ratio of the holes (with constant hole area) was increased. The transmissivity increase was found to be strongly polarization dependent. In addition, the wavelengths at which maximum transmissivity occurs were found to depend on the shape of the holes. The wavelengths at which minima in the transmission occur are the same for all shapes, as expected for Wood's anomaly. Dispersion measurements have shown that for larger angles the influence of the waveguide resonances is minimal and the SPP resonances are the responsible mechanism for the peak wavelengths and intensity of transmissivity spectra.

The dispersion of the main transmission peak is flat for parallel wave vectors close to zero. The range of wave vectors for which the dispersion is flat increases for increasing 
aspect ratio of the holes, indicating an increasingly localized nature of the transmission. Based on this observation and that of the transmission properties of individual holes, we attribute the enhanced transmission to an interaction between surface plasmon resonances at the surface of the metal, on the one hand, and localized resonances inside the holes, on the other hand. This interaction, on the one hand, increases the coupling efficiency between surface plasmons from both sides of the film, resulting is a higher transmissivity. On the other hand, it causes the transmission maxima to be found on different wavelengths for the arrays of which the hole shapes differ. However, our findings show that the contributions of surface plasmons and localized modes to the extraordinary transmission phenomenon cannot be trivially separated in two distinct contributions.

\section{ACKNOWLEDGMENTS}

The work described in this paper is part of and financially supported by the Strategic Research Orientation "Advanced Photonic Structures" of the MESA ${ }^{+}$Research Institute of the University of Twente. This work is part of the research program of the Stichting voor Fundamenteel Onderzoek der Materie (FOM), which is financially supported by the Nederlandse Organisatie voor Wetenschappelijk Onderzoek (NWO).
*Present address: Complex Photonic Systems, MESA ${ }^{+}$Research Institute and Dept. of Science \& Technology, University of Twente, P.O. Box 217, 7500 AE Enschede, The Netherlands.

${ }^{\dagger}$ Electronic address: L.Kuipers@amolf.nl

${ }^{1}$ T. W. Ebbesen, H. J. Lezec, H. F. Ghaemi, T. Thio, and P. A. Wolff, Nature (London) 391, 667 (1998).

${ }^{2}$ T. K. Wu and S. W. Lee, IEEE Trans. Antennas Propag. 42, 1484 (1994).

${ }^{3}$ C. Winnewisser, F. Lewen, J. Weinzierl, and H. Helm, Appl. Opt. 38, 3961 (1999).

${ }^{4}$ H. F. Ghaemi, T. Thio, D. E. Grupp, T. W. Ebbesen, and H. J. Lezec, Phys. Rev. B 58, 6779 (1998).

${ }^{5}$ Q. Cao and P. Lalanne, Phys. Rev. Lett. 88, 057403 (2002).

${ }^{6}$ H. Lezec and T. Thio, Opt. Express 12, 3629 (2004).

${ }^{7}$ L. Martín-Moreno, F. J. García-Vidal, H. J. Lezec, K. M. Pellerin, T. Thio, J. B. Pendry, and T. W. Ebbesen, Phys. Rev. Lett. 86, 1114 (2001).

${ }^{8}$ E. Popov, M. Nevière, S. Enoch, and R. Reinisch, Phys. Rev. B 62, 16100 (2000).

${ }^{9}$ E. Popov, S. Enoch, G. Tayeb, M. Nevière, B. Gralak, and N. Bonod, Appl. Opt. 43, 999 (2004).

${ }^{10}$ W. C. Tan, T. W. Preist, and R. J. Sambles, Phys. Rev. B 62, 11134 (2000).

${ }^{11}$ D. van Labecke, F. I. Baida, and J. M. Vigoureux, J. Microsc. 213, 140 (2004)

${ }^{12}$ W. Fan, S. Zhang, B. Minhas, K. Malloy, and S. Brueck, Phys. Rev. Lett. 94, 033902 (2005).

${ }^{13}$ I. Avrutsky, Y. Zhao, and V. Kochergin, Opt. Lett. 25, 595 (2000).

${ }^{14}$ D. E. Grupp, H. J. Lezec, T. W. Ebbesen, K. M. Pellerin, and T. Thio, Appl. Phys. Lett. 77, 1569 (2000).

${ }^{15}$ T. Thio, H. F. Ghaemi, H. J. Lezec, P. A. Wolff, and T. W. Ebbesen, J. Opt. Soc. Am. B 16, 1743 (1999).

${ }^{16}$ A. Degiron, H. J. Lezec, W. Barnes, and T. Ebbesen, Appl. Phys. Lett. 81, 4327 (2002).

${ }^{17}$ K. L. van der Molen, F. B. Segerink, N. F. van Hulst, and L. Kuipers, Appl. Phys. Lett. 85, 4316 (2004).

${ }^{18}$ D. S. Kim, S. C. Hohng, V. Malyarchuck, Y. C. Yoon, Y. H. Ahn, K. Yee, J. Park, J. Kim, Q. Park, and C. Lienau, Phys. Rev. Lett. 91, 143901 (2003).
${ }^{19}$ H. A. Bethe, Phys. Rev. 66, 163 (1944).

${ }^{20}$ K. J. Klein Koerkamp, S. Enoch, F. B. Segerink, N. F. van Hulst, and L. Kuipers, Phys. Rev. Lett. 92, 183901 (2004).

${ }^{21}$ A. Degiron and T. Ebbesen, J. Opt. A, Pure Appl. Opt. 7, S90 (2005).

${ }^{22}$ R. Gordon, A. G. Brolo, A. McKinnon, A. Rajora, B. Leathem, and K. L. Kavanagh, Phys. Rev. Lett. 92, 037401 (2004).

${ }^{23}$ J. Elliot, I. Smolyaninov, N. Zheludev, and A. V. Zayats, Opt. Lett. 29, 1414 (2004).

${ }^{24}$ J. Elliot, I. I. Smolyaninov, N. I. Zheludev, and A. V. Zayats, Phys. Rev. B 70, 233403 (2004).

${ }^{25}$ H. Cao and A. Nahata, Opt. Express 12, 3664 (2004).

${ }^{26}$ R. W. Wood, Phys. Rev. 48, 928 (1935).

${ }^{27}$ R. Petit, Electromagnetic Theory of Gratings (Springler-Verlag, Berlin, 1980).

${ }^{28}$ M. G. Moharam and T. K. Gaylord, J. Opt. Soc. Am. 71, 811 (1981).

${ }^{29}$ L. Li, J. Opt. Soc. Am. A 14, 2758 (1997).

${ }^{30}$ L. Li, J. Opt. Soc. Am. A 13, 1024 (1996).

${ }^{31}$ M. Nevière and E. Popov, Light Propagation in Periodic Media: Differential Theory and Design (Marcel Dekker, New York, 2003).

${ }^{32}$ D. Maystre, Electromagnetic Modelling of Dielectric and Metallic Photonic Crystals (Wiley, New-York, 2002), Chap. 17, pp. 661-724.

${ }^{33}$ E. D. Palik, Handbook of Optical Constants in Solids (Academic Press, Boston, 1991).

${ }^{34}$ A. V. Zayats and I. Smolyaninov, J. Opt. A, Pure Appl. Opt. 5, S16 (2003).

${ }^{35}$ A. Degiron, H. Lezec, N. Yamamoto, and T. Ebbesen, Opt. Commun. 239, 61 (2004).

${ }^{36}$ R. Gordon and A. Brolo, Opt. Express 13, 1933 (2005).

${ }^{37}$ H. Raether, Surface Plasmons on Smooth and Rough Surfaces and on Gratings (Springer-Verlag, Berlin, 1988).

${ }^{38}$ C. Sönnichsen, T. Franzl, T. Wilk, G. von Plessen, J. Feldmann, O. Wilson, and P. Mulvaney, Phys. Rev. Lett. 88, 077402 (2002)

${ }^{39}$ W. Gotschy, K. Vonmetz, and A. L. abd F. R. Aussenegg, Opt. Lett. 21, 1099 (1996). 